\title{
Dynamic Mechanism of the Ferroelectric to Antiferroelectric Phase Transition in Chiral Smectic Liquid Crystals
}

\author{
Jang-Kun Song, Atsuo Fukuda, and J. K. Vij* \\ Department of Electronic and Electrical Engineering, Trinity College, University of Dublin, Dublin 2, Ireland
} (Received 21 December 2007; revised manuscript received 16 June 2008; published 28 August 2008)

\begin{abstract}
The temperature-induced phase transition between the chiral smectic phases, antiferroelectric (smectic- $C_{A}^{*}$ ) and ferroelectric (smectic- $C^{*}$ ), is found to occur through solitary wave propagation. We measure the free energy, which shows a double well shape in the entire $\operatorname{Sm} C_{A}^{*}$ temperature range and the global minimum is found to shift from the antiferroelectric order to the ferroelectric order at the transition temperature. However, any significant supercooling is not observed and the transition cannot be described by the first order Landau-de Gennes theory, where the double well potential exists only in a narrow range of temperatures. This implies that the $\mathrm{Sm} C_{A}^{*}-\mathrm{Sm} C^{*}$ transition can occur only nonhomogeneously through the solitary wave propagation which overcomes the high energy barrier between the two minima.
\end{abstract}

Liquid crystals have played an important role in clarifying the various aspects of phase transitions in condensed matter physics due to the existence of a variety of phases. In this Letter, we report and clarify an important characteristic feature of the temperature-induced phase transition between the $\mathrm{SmC}_{A}^{*}$ and $\mathrm{SmC} C^{*}$ phases for the first time. We observe the temperature-induced solitary wave propagation that brings about the $\mathrm{Sm} C_{A}^{*}$-Sm $C^{*}$ phase transition and find from the observations that the $\mathrm{Sm} C^{*}$ free energy minimum persists in the entire $\mathrm{Sm}_{A}^{*}$ temperature range. At the same time, significant supercooling is not observed at the transition. Based on the knowledge that the established tilted smectic structure almost completely suppresses the azimuthal-angular thermal motion of liquid crystalline molecules, it is simply expected that the free energy barrier hinders the transition from the local $\mathrm{Sm} C^{*}$ minimum to the global $\mathrm{Sm} C_{A}^{*}$ minimum even below the critical temperature. As actually observed here, however, the elastically coupled liquid crystalline molecular motions overcome the free energy barrier by forming propagating solitary waves [1].

Tilted smectic phases have a multilayered structure that usually consists of a number of single-molecular layers. The average molecular direction in a layer, i.e., the in-layer director, is slanted from the layer normal by the tilt angle $\theta$, which is constant over all layers, and is represented as $\mathbf{n}_{i}=$ $\left(n_{i x}, n_{i y}, n_{i z}\right)=\left(\sin \theta \cos \varphi_{i}, \sin \theta \sin \varphi_{i}, \cos \theta\right)$ for the $i$ th layer in the coordinate system shown in Fig. 1. The azimuthal angle difference, $\phi\left(\equiv \varphi_{i+1}-\varphi_{i}\right)$, between the two neighboring layers can be either synclinic (i.e., $\phi \sim 0$ ) or anticlinic (i.e., $\phi \sim \pi$ ) as in $\mathrm{SmC}^{*}$ and $\mathrm{Sm}_{A}^{*}$ phases, respectively $[2,3]$. Hence, the transitions between these phases occur through a change of $\phi$ between $\phi \sim 0$ and $\phi \sim \pi$. The phase transitions between the tilted smectic phases have been explained using various phenomenological models, most of which are based on the Landaude Gennes theory. The first phenomenological model regarding the $\mathrm{SmC}_{A}^{*}$-SmC ${ }^{*}$ phase transition was sug- gested by Orihara and Ishibashi [4]. They introduced a modified director vector, $\xi_{i}=\left(-n_{i y} n_{i z}, n_{i x} n_{i z}\right)=$ $\sin \theta \cos \theta\left(-\sin \varphi_{i}, \cos \varphi_{i}\right)$, and chose $\xi_{s}=\left(\xi_{i}+\xi_{i+1}\right) / 2$ and $\xi_{a}=\left(\xi_{i}-\xi_{i+1}\right) / 2$ for the order parameters in the Landau-de Gennes theory. Here, $\xi_{s}$ and $\xi_{a}$ are maximized at the synclinic and the anticlinic orderings, respectively. They allowed the variation of $\theta$ as well as of $\varphi$ from layer to layer, but the variation of $\theta$ has been ruled out by the successive experimental observations [5]. Consequently, the free energy simply becomes a function of $\phi\left(\equiv \varphi_{i+1}-\right.$ $\left.\varphi_{i}\right)$ at a fixed temperature as
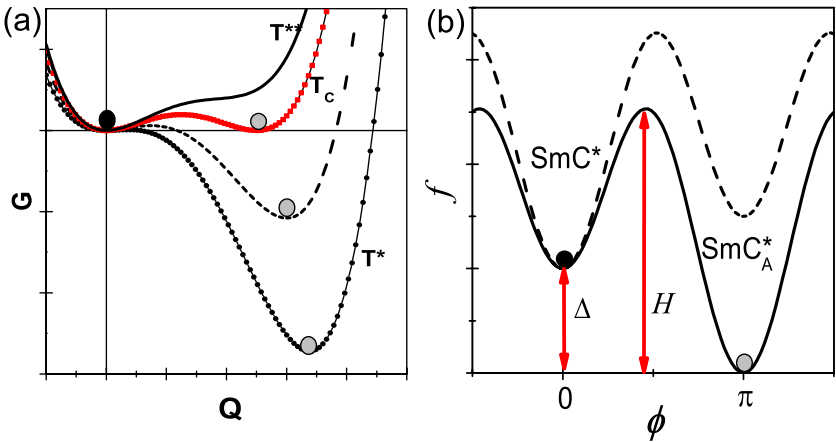

(c)
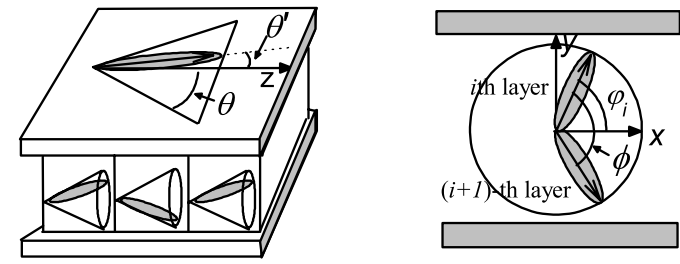

FIG. 1 (color online). (a) Landau-de Gennes free energy $G$ as a function of order parameter $Q$ for different temperatures (b) $f$ as a function of $\phi$ in $\mathrm{SmC}_{A}^{*}$ (solid line) and in $\mathrm{SmC}^{*}$ (dashed line). (c) Schematics of a planar unwound $\mathrm{Sm} C_{A}^{*}$ cell, triangle on top of glass is a projection of the smectic cone. The apparent tilt angle $\theta^{\prime}$ under electric field is distinguished from the phase tilt angle $\theta$, where molecules are aligned vertically $[11,12]$. 


$$
\begin{aligned}
f= & \alpha_{a} \xi_{a}^{2}+\alpha_{s} \xi_{s}^{2}+\beta_{a} \xi_{a}^{4}+\beta_{s} \xi_{s}^{4}+\gamma \xi_{a}^{2} \xi_{s}^{2} \\
= & A\left(\alpha_{s}+\alpha_{a}\right)+\left(A^{2} / 2\right)\left(3 \beta_{s}+3 \beta_{a}+\gamma\right) \\
& +\left\{A\left(\alpha_{s}-\alpha_{a}\right)+2 A^{2}\left(\beta_{s}-\beta_{a}\right)\right\} \cos \phi \\
& +\left(A^{2} / 2\right)\left(\beta_{s}+\beta_{a}-\gamma\right) \cos 2 \phi \\
\equiv & C+D \cos \phi-Q \cos 2 \phi,
\end{aligned}
$$

where $A\left(\equiv\left\{\sin ^{2}(2 \theta)\right\} / 8\right), C, D$, and $Q$ are constants at a fixed temperature. Thus, the free energy for the $\mathrm{SmC}_{A}^{*}-\mathrm{Sm} C^{*}$ phase transition can be expressed as the interlayer interaction energy between the neighboring layers consisting of the dipolar $(\propto \cos \phi)$ and the quadrupolar $(\propto \cos 2 \phi)$ terms. Recent phenomenological models basically include these terms, and these are added to other terms [6-9]. In fact, this interlayer interaction energy form has been suggested intuitively and used in analyzing the electric-field-induced $\mathrm{SmC}_{A}^{*}$-SmC $C^{*}$ switching [10-12], though not used for explaining the phase transition.

Figure 1 shows a comparison of the two methods: (a) the weakly first order transition described by the Landaude Gennes theory and (b) the interlayer interaction described by Eq. (1). The free energy difference $\Delta$ and the barrier height $H$ between the $\mathrm{SmC}_{A}^{*}$ and $\mathrm{SmC}^{*}$ minima illustrated in Fig. 1 are calculated by using Eq. (1),

$$
\Delta=2 D, \quad H=\left\{D^{2} /(8 Q)\right\}+2 Q+|D| .
$$

Recently, we devised and reported a new method for determining $D$ and $Q$ experimentally in the antiferroelectric cell [12]. During the field-induced switching in the antiferroelectric cell, the solitary wave propagates as $\mathrm{Li}$ et al. [13] actually observed and Song et al. extended and modeled it appropriately [12]. The model allows us to measure $D$ and $Q$. Here, the method is briefly explained. The free energy of a cell having a solitary wave propagating along the $x$ axis under the application of an electric field $E$ can be obtained by adding the elastic energy of the soliton and the coupling energy of the spontaneous polarization $P_{s}$ with $E$ into Eq. (1) as

$$
\begin{aligned}
f^{\prime}= & K^{\prime}\left(\partial \varphi_{i} / \partial x\right)^{2}-P_{s} E \cos \phi \\
& +D \cos \phi-Q \cos 2 \phi+C \\
\equiv & K^{\prime}\left(\partial \varphi_{i} / \partial x\right)^{2}+U(\phi),
\end{aligned}
$$

where the effective elastic coefficient $K^{\prime}=K \sin ^{2}(\theta) / 2$. The first and second terms represent elastic and electric coupling energies, respectively. The kinetics of the soliton is governed by the total potential energy $U$. To have the solitary wave, $U$ should have two energy minima, where $\phi=0$ for the synclinic minimum and $\phi \approx \pi$ for the anticlinic minimum. At the threshold field $E_{\mathrm{th}}$, the solitary wave has a zero speed, and the potential energy at the two minima becomes the same. Hence,

$$
\begin{gathered}
U\left(E=E_{\mathrm{th}}, \phi=0\right)=U\left(E=E_{\mathrm{th}}, \phi=\phi_{\mathrm{th}}\right), \\
(\partial U / \partial \phi)_{\phi=0}=(/ \partial U / \partial \phi)_{\phi=\phi_{\mathrm{th}}}=0,
\end{gathered}
$$

where $\phi_{\mathrm{th}}$ is the value of $\phi$ at $E=E_{\mathrm{th}}$. From Eqs. (4) and (5), we can find that

$$
\begin{gathered}
D=\frac{3 \cos \left(\phi_{\mathrm{th}} / 2\right)+2}{4\left\{\cos \left(\phi_{\mathrm{th}} / 2\right)+1\right\}^{2}} P_{s} E_{\mathrm{th}}, \\
Q=\frac{1}{16 \cos \left(\phi_{\mathrm{th}} / 2\right)\left\{\cos \left(\phi_{\mathrm{th}} / 2\right)+1\right\}^{2}} P_{s} E_{\mathrm{th}} .
\end{gathered}
$$

We can determine $\phi_{\text {th }}=\pi-2 \sin ^{-1}\left(\tan \theta^{\prime} / \tan \theta\right)$ by measuring the apparent tilt angle $\theta^{\prime}$ at $E_{\text {th }}$ and the phase tilt angle $\theta$ shown in Fig. 1. Hence, $D$ and $Q$ are determined experimentally.

The compounds used are MC-513 and MC-881 synthesized by the Mitsubishi Gas Chemical Company, Inc. [14]. The phase sequence of $\mathrm{MC}-513$ is Cry. $\left(38^{\circ} \mathrm{C}\right) \mathrm{Sm} C_{A}^{*}$ $\left(73^{\circ} \mathrm{C}\right) \mathrm{SmC}^{*}\left(81^{\circ} \mathrm{C}\right) \operatorname{Sm} A\left(85^{\circ} \mathrm{C}\right)$ I. Having a wide temperature range of $\mathrm{Sm} C_{A}^{*}$ and $\mathrm{Sm} C^{*}, \mathrm{MC}-513$ is particularly suited for demonstrating the phenomenon under consideration. For comparison, MC-881 is used, the phase
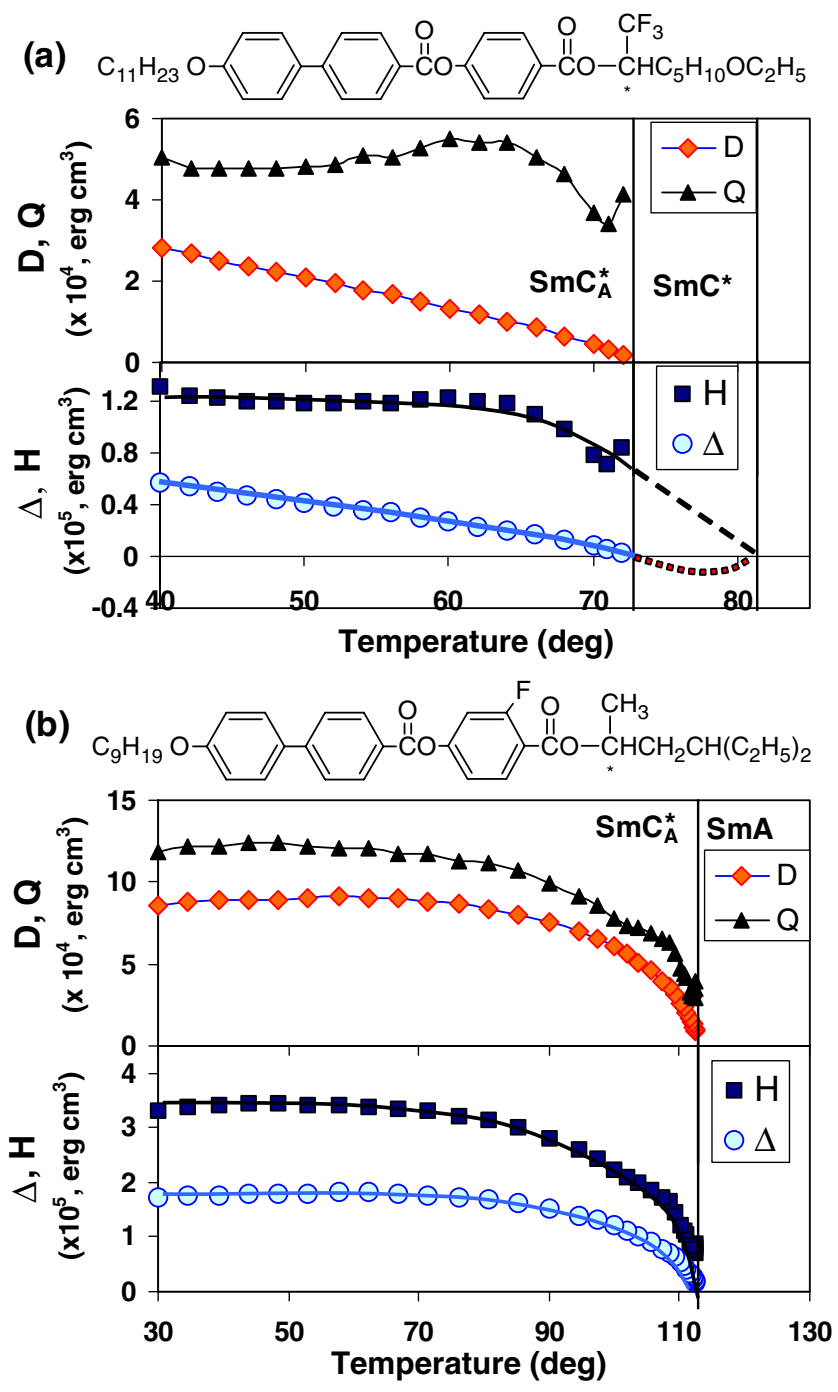

FIG. 2 (color online). Experimentally obtained $D, Q, \Delta$, and $H$ for MC-513 (a) and MC-881 (b). The experimental data of MC881 were taken from Ref. [12]. 
sequence of which is $\operatorname{SmC}_{A}^{*}\left(112.5^{\circ} \mathrm{C}\right) \operatorname{Sm} A\left(120^{\circ} \mathrm{C}\right) \mathrm{I}$. MC-881 does not exhibit $\mathrm{SmC}^{*}$. Consequently, the $\mathrm{SmC}_{A}^{*}-\mathrm{Sm} C^{*}$ phase transition is missing. It is therefore suitable for drawing a comparison with MC-513 with a $\mathrm{SmC}_{A}^{*}$ to $\mathrm{Sm} C^{*}$ transition.

Figure 2 shows $D, Q, \Delta$, and $H$ calculated from $P_{s}, E_{\mathrm{th}}$, $\theta$, and $\theta^{\prime}$. These were measured in a homogeneously aligned $25 \mu \mathrm{m}$ thick cell for MC-513 and data for MC881 were taken from our previous paper [12]. $Q$ is found to be roughly proportional to the fourth power of $\theta$. The parameters $D, Q, \Delta$, and $H$ must become zero at the phase transition temperature to $\operatorname{Sm} A$ as is actually confirmed in MC- 881 and anticipated by extrapolation [the dashed and dotted lines of Fig. 2(a)] in MC-513. This is not clear in MC-513 where we could not obtain $\Delta$ and $H$ in $\mathrm{SmC}^{*}$. However, simple extrapolation indicates that $\Delta$ varies gradually and changes its sign at the $\mathrm{SmC}_{A}^{*}-\mathrm{Sm} C^{*}$ transition temperature, which is the same behavior as in the Landau-de Gennes theory for the weakly first order phase transition. On the other hand, $H$ has large positive value over the entire temperature range of smectic- $\mathrm{C}$ phases, which exhibits a striking contrast to the weakly first order phase transition frequently encountered in liquid crystals and treated by the Landau-de Gennes theory $[15,16]$, where we define two additional characteristic temperatures $T^{*}$ and $T^{* *}$ close to the critical temperature $T_{\mathrm{c}}$ as shown in Fig. 1. On cooling, $T^{*}$ is the limit of metastability of the high temperature phase; its free energy still shows a local minimum though it is not a global minimum for $T^{*}<T<$ $T_{\mathrm{c}}$. The limit of metastability of the low temperature phase occurs at $T^{* *}$, where the secondary minimum disappears on heating. The obtained large $H$ implies that the free energy has always two minima separated by the barrier $H$ in the entire temperature range of $\mathrm{SmC}_{A}^{*}$ and $\mathrm{Sm} C^{*}$. The free energy does not have temperatures $T^{*}$ and $T^{* *}$ as in Fig. 1(a) under the Landau-de Gennes theory for the first order phase transition. Hence, very large supercooling and superheating could be expected, or $\mathrm{Sm} C_{A}^{*}$ may not appear at all due to the high energy barrier between the synclinic and the anticlinic minima while cooling. However, the supercooling or superheating observed at the $\mathrm{Sm}_{A}^{*}$-SmC $C^{*}$ phase transition is actually less than a few degrees if the effects due to the substrate interfaces are minimized.

Two fundamental questions arise: How does the phase transition overcome the free energy barrier between the two states, and why is the supercooling or superheating not large? The solitary wave propagation [1], which is a consecutive transition of one state to another state as dominoes, can give a clue for answering the questions. The solitary wave in itself is quite an interesting physical phenomenon. This is a solution of a nonlinear equation, with a property that energy is transmitted in a localized way and occurs in diverse phenomena in physics, such as in ocean as Tsunami, string theory as $D$-branes, and biology where these occur as Davidoff solution for modeling ad- enosine triphosphate (ATP). In liquid crystals, the solitary waves were observed in the nematic-SmA transition [17] and in the switching of the surface stabilized ferroelectric liquid crystal cell [18] and in the switching of the $\operatorname{Sm} C_{A}^{*}$ cell [13].

Likewise, in the temperature-induced phase transition under consideration where $\Delta$ varies gradually to zero and $H$ remains still large, the temperature-induced solitary wave propagation is naturally expected. To explain the solitary wave, we start from Eq. (3) by inserting $E=0$, because we are considering the intrinsic phase transition but not the field-induced switching. The corresponding EulerLagrange equation for the solitary wave is written as $[1,12]$

$$
\gamma \frac{\partial \phi}{\partial t}-K^{\prime} \frac{\partial^{2} \phi}{\partial x^{2}}=-\frac{d U(\phi)}{d \phi}=D \sin \phi-2 Q \sin 2 \phi .
$$
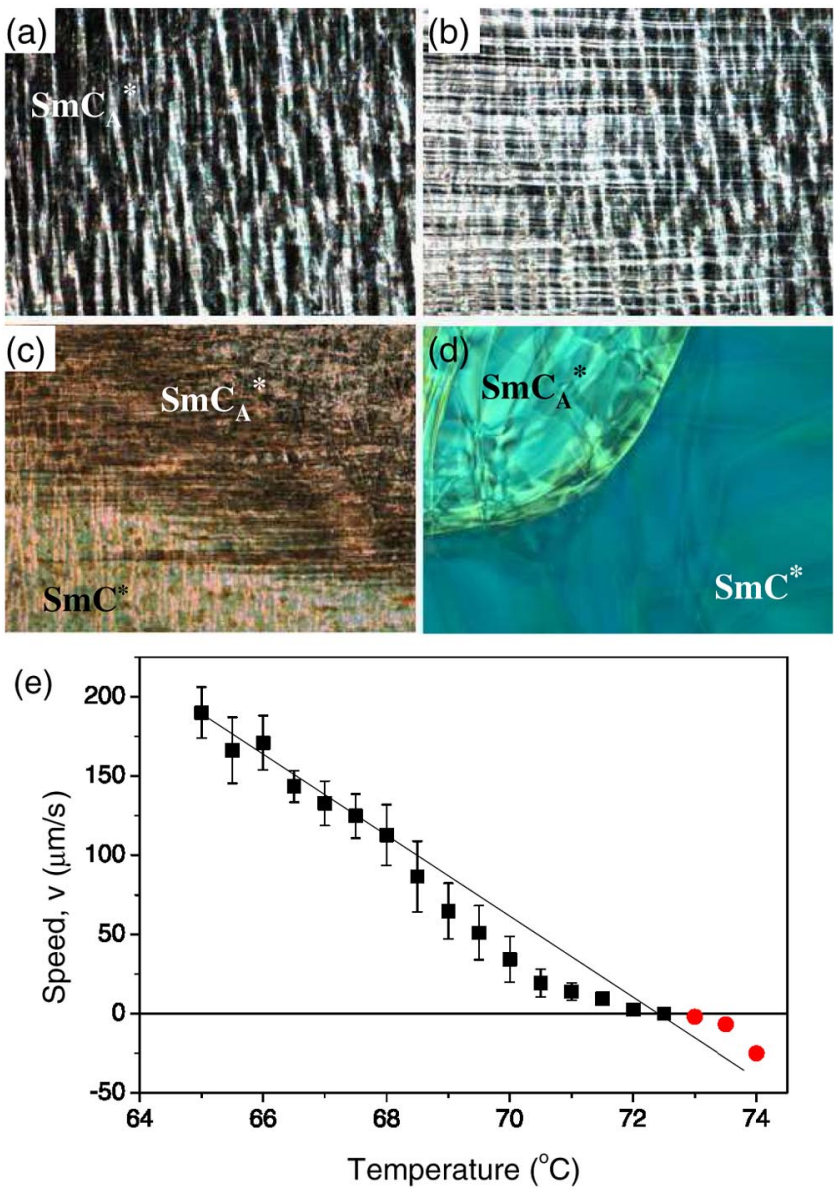

FIG. 3 (color online). (a)-(d) Micrographs taken under the two polarizers, the angle between which was selected for the sharpest distinction between $\mathrm{SmC}^{*}$ and $\mathrm{SmC}_{A}^{*}$, and (e) the speed of solitary wave as a function of temperature. Images were taken (a) immediately after heating an isothermal cell slightly higher than $T_{c}$, (b) after $10 \mathrm{~s}$, (c) at the phase transition in a temperature gradient cell with a $1.3^{\circ} \mathrm{C}$ temperature difference between the left bottom and right top, and (d) during slow cooling from $\mathrm{SmC}^{*}$ in a free standing film, where many propagating domain walls were observed. 
The physically interesting solution of Eq. (8) is

$$
\phi(x)=\tan ^{-1} e^{-(x-v t) / d},
$$

where

$$
\begin{gathered}
d=\frac{1}{4} \sqrt{\frac{K^{\prime}}{Q}} \simeq \frac{1}{2 \sqrt{2}} \sqrt{\frac{K^{\prime}}{H}}, \\
v=\frac{4 d D}{\gamma}=\frac{2 d \Delta}{\gamma} \simeq \frac{s_{0}}{\sqrt{2} \gamma} \sqrt{\frac{K^{\prime}}{H}}\left(T_{c}-T\right),
\end{gathered}
$$

where $\gamma$ is the viscosity of the medium, and $d$ and $v$ are the width and the speed of the solitary wave. Here we use linear approximation for $\Delta$ near $T_{c}$ so that $\Delta \simeq s_{0}\left(T_{c}-T\right)$, where $s_{0}$ is the slope of $\Delta$. The width of the solitary wave is inversely proportional to $\sqrt{H}$; the speed of the solitary wave, also inversely proportional to $\sqrt{H}$, is proportional to $T_{c}-T$ and hence changes its sign at the phase transition temperature.

For observations of the solitary wave, we made a $25 \mu \mathrm{m}$ thick homogeneously aligned (planar) cell of MC-513. This large cell thickness avoids the excessive surface effect from the alignment layer. Figures 3(a) and 3(b) show the temperature-induced solitary waves, which are observed for the first time as the temperature-induced $\mathrm{SmC}_{A}^{*}$-SmC $C^{*}$ phase transition. The cell was kept at $71^{\circ} \mathrm{C}$ while in $\mathrm{Sm} C_{A}^{*}$. Then we increased the temperature to $73.5^{\circ} \mathrm{C}$, which is slightly higher than $T_{c}$. When the temperature reaches this value, the image in Fig. 3(a) is taken while still in $\mathrm{SmC}_{A}^{*}$. The solitary waves, which consist of a number of lines parallel to the layer plane, start to propagate as shown in Fig. 3(b). The solitary waves propagate in the reverse direction when the temperature is decreased below the transition temperature, as expected from Eq. (11). Next, we made a temperature gradient cell by inserting a thin insulator between the heater and one of the sides of the cell. The temperature difference across the two edges in the microscopic images is found to be $\sim 1.3{ }^{\circ} \mathrm{C}$. As shown in Fig. 3(c), the boundary of the $\mathrm{SmC}_{A}^{*}$-SmC $C^{*}$ transition runs zigzag, which is of similar shape observed in the electricfield-induced solitary wave $[12,13]$. The propagating solitary waves are observed in a free standing film almost free from the surface effect as shown in Fig. 3(d). The measured speed of the solitary wave is given in Fig. 3(e). For $T<T_{c}$, speed is measured at zero field by first inducing the $\mathrm{SmC}^{*}$ domain by applying the field, and then turning it off. However, for $T>T_{c}$, neither the $\mathrm{SmC}_{A}^{*}$ domain nor the domain walls can be obtained. Therefore, by heating the cell quickly from just below to slightly above $T_{c}$, the speed of the domain wall is measured. As shown in Fig. 3, the measured speed of the solitary wave shows rather linear dependence with temperature. The slight mismatch near the phase transition temperature may be caused by the frictional effect of surfaces, which is interesting but beyond our discussion here.

To summarize, we experimentally obtain the free energy difference $\Delta$ and the barrier height $H$ between $\operatorname{Sm}_{A}^{*}$ and
$\mathrm{SmC}^{*}$ minima. It is of double well shape in the entire range of temperatures of the $\mathrm{SmC}_{A}^{*}$ phase, which is a surprising and interesting phenomenon. The transition occurs only through a nonhomogeneous process; i.e., the solitary wave propagation overcomes the high free energy barrier. The mechanism is applicable not only for the $\mathrm{SmC}_{A}^{*}-\mathrm{Sm} C^{*}$ transition but may also be applicable for the phase transitions between all the smectic $C$ variant phases [6]. It means that the intrinsic phase transitions between these phases are actually strong first order transitions, but the propagating solitary waves make these transitions have the properties of weakly first order. We observe the propagating solitary waves through a microscope, and measure the speed as a function of temperature. Basically, the solitary wave can arise from defects or imperfect boundaries. Therefore, if we make a cell that satisfies the perfect condition of no imperfections, we may observe very large supercooling and may even cool down the cell to the crystalline phase without observing $\operatorname{Sm} C_{A}^{*}$. We can also explain the strong dependence of the phase transition temperature on the cell thickness.

We thank SFI for funding the research, and Mitsubishi Gas Chemical Company, Inc. for LC materials. J. K. S. thanks Samsung Electronics Co., Ltd. for financial support.

*jvij@tcd.ie

[1] E. Magyari, Phys. Rev. B 29, 7082 (1984).

[2] A. D. L. Chandani, E. Gorecka, Y. Ouchi, H. Takezoe, and A. Fukuda, Jpn. J. Appl. Phys. 28, L1265 (1989).

[3] R. B. Meyer, L. Liebert, L. Strzelecki, and P. Keller, J. Phys. (Paris), Lett. 36, L69 (1975).

[4] H. Orihara and Y. Ishibashi, Jpn. J. Appl. Phys. 29, L115 (1990).

[5] P. Cluzeau et al., Mol. Cryst. Liq. Cryst. 260, 69 (1995).

[6] A. V. Emelyanenko, A. Fukuda, and J. K. Vij, Phys. Rev. E 74, 011705 (2006).

[7] A. Roy and N. V. Madhusudana, Europhys. Lett. 36, 221 (1996).

[8] M. A. Osipov and A. Fukuda, Phys. Rev. E 62, 3724 (2000).

[9] M. Cepic and B. Zeks, Phys. Rev. Lett. 87, 085501 (2001).

[10] T. Qian and P. L. Taylor, Phys. Rev. E 60, 2978 (1999).

[11] L. A. Parry-Jones and S. J. Elston, Appl. Phys. Lett. 79, 2097 (2001).

[12] J. K. Song, A. Fukuda, and J. K. Vij, Phys. Rev. E 76, 011708 (2007).

[13] J. Li, X. Wang, E. Kangas, P. L. Taylor, C. Rosenblatt, Y. Suzuki, and P. E. Cladis, Phys. Rev. B 52, R13075 (1995).

[14] T. Matsumoto et al., J. Mater. Chem. 9, 2051 (1999).

[15] P. G. de Gennes and J. Prost, The Physics of Liquid Crystals (Clarendon Press, Oxford, 1993), Chap. 10.

[16] P. M. Chaikin and T.C. Lubensky, Principles of Condensed Matter Physics (Cambridge University Press, Cambridge, England, 1995), Chap. 4.

[17] P. E. Cladis et al., Phys. Rev. Lett. 62, 1764 (1989).

[18] M. A. Handschy and N. A. Clark, Appl. Phys. Lett. 41, 39 (1982). 